\title{
A Reconfigurable Coplanar Waveguide Bowtie Antenna Using an Integrated Ferroelectric Thin-Film Varactor
}

\author{
K. C. Pan, ${ }^{1}$ D. Brown, ${ }^{1}$ G. Subramanyam, ${ }^{1}$ R. Penno, ${ }^{1}$ H. Jiang, ${ }^{1}$ C. H. Zhang, ${ }^{1}$ \\ M. Patterson, ${ }^{1}$ D. Kuhl, ${ }^{2}$ K. Leedy, ${ }^{2}$ and C. Cerny ${ }^{2}$ \\ ${ }^{1}$ Department of Electrical and Computer Engineering, University of Dayton, 300 College Park, Dayton, OH 45469, USA \\ ${ }^{2}$ Air Force Research Lab, Sensors Directorate, WPAFB, Dayton, OH 45432, USA
}

Correspondence should be addressed to D. Brown, brownd7@udayton.edu

Received 10 March 2012; Revised 11 June 2012; Accepted 17 June 2012

Academic Editor: Hala A. Elsadek

Copyright ( $\odot 2012$ K. C. Pan et al. This is an open access article distributed under the Creative Commons Attribution License, which permits unrestricted use, distribution, and reproduction in any medium, provided the original work is properly cited.

A novel printed antenna with a frequency reconfigurable feed network is presented. The antenna consists of a bowtie structure patch radiating element in the inner space of an annulus that is on a nongrounded substrate with a ferroelectric (FE) Barium Strontium Titanate (BST) thin film. The bowtie patch is fed by a coplanar waveguide (CPW) transmission line that also includes a CPW-based BST shunt varactor. Reconfiguration of the compact $8 \mathrm{~mm} \times 8 \mathrm{~mm}$ system has been demonstrated by shifting the antenna system's operating frequency $500 \mathrm{MHz}$ in the 7-9 GHz band by applying a DC voltage bias.

\section{Introduction}

Because many antennas are narrowband in frequency, more than one antenna is usually needed in systems that operate over multiple frequency bands. Mobile phones and other portable communication devices, for example, are beginning to require broader bandwidths to support their numerous applications and communication protocols. Due to this growing spectrum usage in single devices, more versatile or reconfigurable antennas are desirable to reduce the total required parts as well as overall cost.

The purpose of this paper is to discuss the design of a reconfigurable CPW feed network for a single-layer-patch antenna. Previously presented microstrip patch antenna designs [1] utilized a thin-film ferroelectric (FE) barium strontium titanate (BST) layer and varactor [2] to the feed line. The system presented here improves upon this design by integrating the above varactor with a CPW-based antenna. By using the same transmission line architecture, the fabrication process is simplified and impedance matching between the two devices is much more straightforward.

An added benefit to using BST is that the film has a high dielectric constant, $\varepsilon_{r}$. This material attribute reduces the physical wavelength, thus allowing the antenna to be more compact compared to an antenna on more traditional dielectric substrates.

The paper discusses the design of the antenna, electromagnetic simulation of the antenna. Experimental results of the antenna's impedance matching bandwidth and radiation performance are also be presented.

\section{Antenna Design}

As the overall purpose of this paper is to have an antenna system capable of reconfigurability, the design process was first approached by addressing a novel means of reconfiguration. As antennas and the rest of the RF chain typically require matching networks to allow for maximum power transfer, it was proposed that having a flexible, lumped element device close to the feed of an antenna would allow for a more versatile system.

By the addition of a shunt varactor to the feed network, the combination of which can be depicted by Figure 1, the antenna system's impedance can be adjusted. This reconfigurable matching network can be tuned based on the frequency band of interest. Looking at the admittance of the 
antenna, transmission feed, and varactor, the input admittance of the system can be expressed by

$$
\begin{gathered}
Y_{11}=Y_{\text {in }}=Y_{\text {in } 1}+j \omega C, \\
Y_{\text {in } 1}=\frac{1}{Z_{\text {in } 1}}=\frac{1}{Z_{o}}\left[\frac{Z_{o}+j Z_{A} \tan \beta L_{6}}{Z_{A}+j Z_{o} \tan \beta L_{6}}\right],
\end{gathered}
$$

where $Z_{A}$ is the antenna impedance and the $C$ term is the capacitance of the shunt varactor. As this capacitance is adjustable, the system can thus be reconfigured to provide an ideal match across a wider range of frequencies.

The shunt thin-film device described in [2] provides a unique, thin-film varactor that can be used in the impedance matching network of the antenna system. With this particular device, the capacitance in the admittance equation above is a function of the applied bias voltage. The varactor is implemented using the four-layer structure shown in Figure 2.

As the thin-film varactor described in [2] was decided upon as the method of reconfiguration, requirements of the antenna's design and shape were defined in order to properly integrate with the varactor. First, the varactor is designed to work with a transmission line characteristic impedance of 50 ohms, so this was also the desired characteristic impedance of the designed antenna feed line. Next, to avoid losses from changing transmission line types, the antenna was designed to also have the same transmission line architecture as the varactor. As the varactor utilizes a $\mathrm{CPW}$ transmission line, this meant the antenna would involve surrounding the radiating element by a ground plane. This requirement was also selected as CPW-based antennas have been demonstrated as having low radiation loss [3]. Finally, the varactor utilizes both the bulk substrate and the thin-film BST dielectric. As the antenna will be a patch using one metal layer, the antenna will be designed to operate above both dielectrics with the radiator residing above the BST thin film.

It is desirable to have a large tuning range for the varactor to have the largest degree of reconfiguration in the resulting system. The dielectric constant, $\varepsilon_{r}$, of the BST is demonstrated having tunability of $4: 1$ max-to-min ratio for a thickness of $0.25 \mu \mathrm{m}$ [4-6] on sapphire substrates. To leverage this versatility, the intended wafer for this design was decided to be a $600 \mu \mathrm{m}$ thick sapphire substrate, with a $0.25 \mu \mathrm{m}$ BST layer deposition.

Traditional bowtie antennas are known for large bandwidths while utilizing a single metallization layer, providing distinct advantages during fabrication [7]. This bandwidth was a desirable trait when designing the reconfigurable system as the goal was to achieve a wide operating range. For this reason a bowtie was selected as the basis of the radiating element for the reconfigurable system.

Starting from a CPW bowtie shape, antenna models were designed and simulated in the commercial microwave frequency simulation tool AWR. As the varactor used in the feed line loading was previously created in this software environment, creation of the resulting system involved only the design of the antenna. In an effort to constrain the resulting size of the system on the sapphire wafer, the target frequency range was selected to be C-Band. Antenna shape

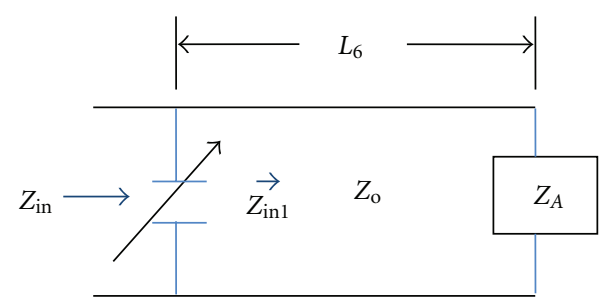

FIgURE 1: Model of the antenna system including CPW feed line and shunt varactor.

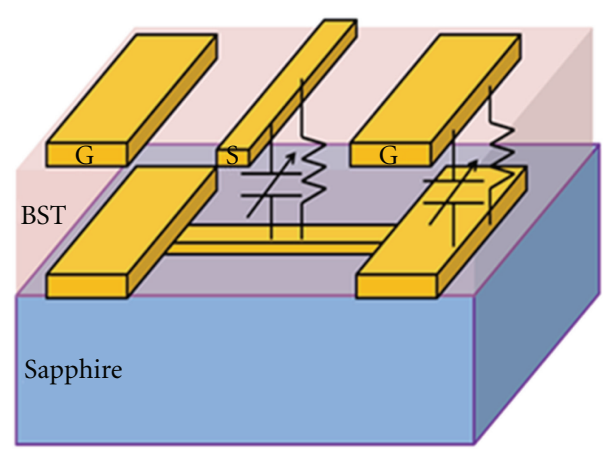

FIGURE 2: BST thin-film varactor structure.

and dimensions were revised incrementally until simulated performance met the desirable frequency bandwidth performance.

The resulting antenna system is shown in Figure 3. The varactor is integrated in front of the transmission line that feeds the CPW bowtie patch antenna. As can be seen, the actual radiating elements are $4.8 \mathrm{~mm} \times 5.1 \mathrm{~mm}$, or $0.12 \lambda_{o} \times$ $0.1275 \lambda_{o}$, where $\lambda_{o}$ is the free space wavelength. The overall size of the antenna system is $8 \mathrm{~mm} \times 8 \mathrm{~mm}$ which is approximately $0.2 \lambda_{o} \times 0.2 \lambda_{o}$ which also shows the entire system works at dimensions that are fractions of the free space wavelength.

\section{Testing Methodology}

To analyze the performance of the antenna system, the frequency swept scattering parameters ( $S$ Parameters) were simulated using the AWR electromagnetic model and also measured on the fabricated device. The fabricated device also underwent testing in an anechoic chamber to analyze radiated fields produced by the antenna.

The $S$ parameters of the fabricated antenna system were measured using an HP8720B vector network analyzer. An on-wafer probe station was fitted with a CASCADE SPACP40-GSG-150-C CPW probe for measurements. To test the tuning performance of the system, a Keithly 2400 source meter was used to provide the DC biasing voltage. The system was tested with biasing voltages ranging from 0 to 10 volts.

The far-field radiation patterns of the reconfigurable antenna under 0 volt bias voltage conditions were measured in the Radiation and Scattering Compact Antenna Laboratory (RASCAL) at the Air Force Research Laboratory. 




\begin{tabular}{|l|l|}
\hline Name & Length $(\mu \mathrm{m})$ \\
\hline$L_{1}$ & 8000 \\
\hline$L_{2}$ & 5550 \\
\hline$L_{3}$ & 4800 \\
\hline$L_{4}$ & 4100 \\
\hline$L_{5}$ & 200 \\
\hline$L_{6}$ & 4600 \\
\hline$L_{7}$ & 600 \\
\hline$W_{1}$ & 8000 \\
\hline$W_{2}$ & 500 \\
\hline$W_{3}$ & 2300 \\
\hline$W_{4}$ & 150 \\
\hline$W_{5}$ & 1300 \\
\hline$W_{6}$ & 200 \\
\hline$W_{7}$ & 3550 \\
\hline
\end{tabular}

Figure 3: A CPW-Fed bowtie slot antenna connected with a BST thin-film varactor.

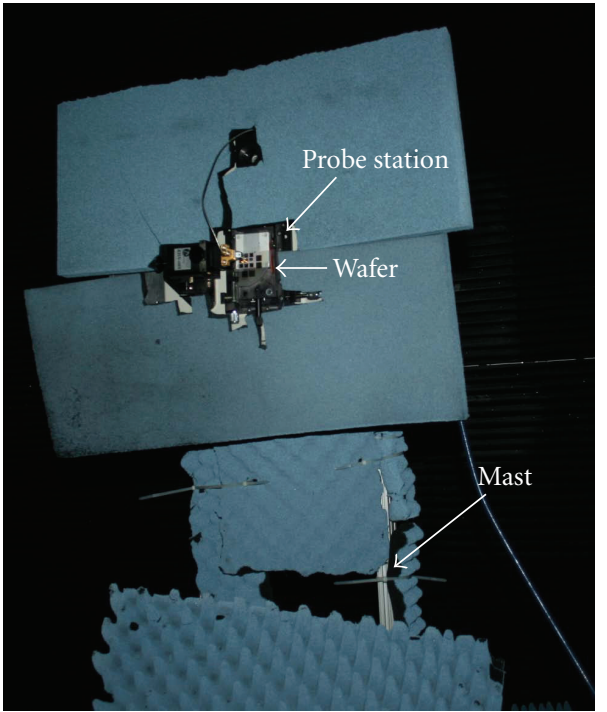

FIGURE 4: Radiation measurement setup in the RASCAL anechoic chamber.

To measure the far-field radiation patterns, the reconfigurable antenna was turned mechanically in an anechoic chamber. A rotating mast was in the center of the chamber, with a portable probe station connected to it. The probe station was used to hold the antenna as shown in Figure 4. The magnitude and phase data was collected and measured for the various angles in the mechanical sweep.

\section{Results and Discussions}

Plots of the simulated and measured system performance can be seen in Figures 5-9. Electromagnetic simulations of the antenna with the CPW feed line were run both with and

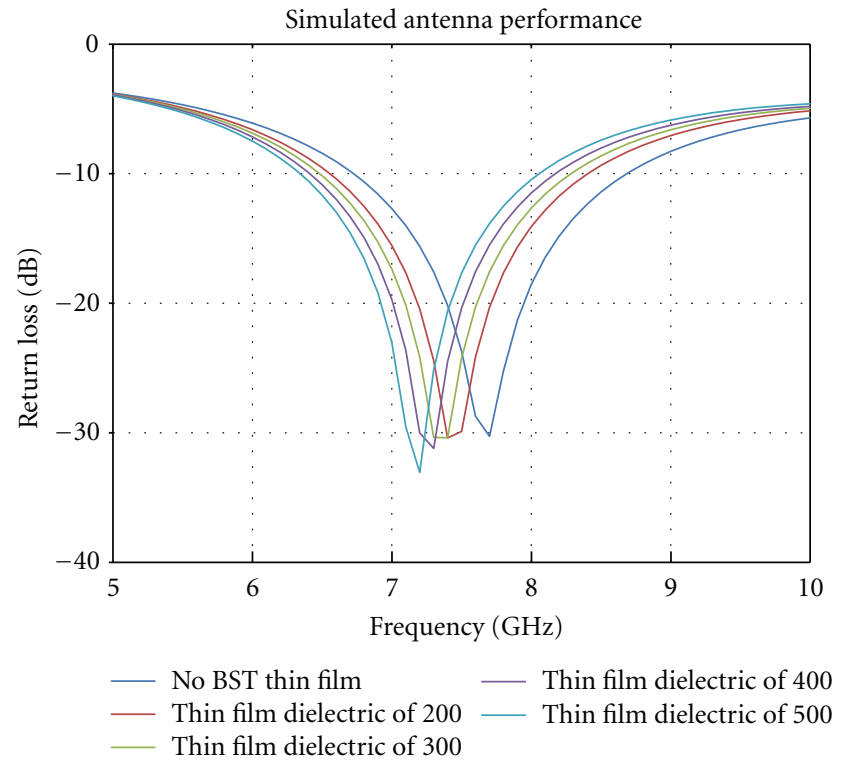

FIGURE 5: Bowtie antenna on different BST permitivities.

without a varactor connected in the AWR environment to show how the system changes with a varactor present.

Figure 5 shows the simulated results of the CPW patch antenna atop BST thin films of varying permittivity values as well as a reference design of the antenna above only the bulk sapphire. From the figure it is seen that the antenna undergoes a miniaturization, as shown by the notch point moving to lower frequency when above BST. The permittivity numbers simulated in this comparison also agree with those permittivity numbers from the tuning of previously published research [2].

Figures 6 and 8 show the electromagnetic simulation results of the antenna system under tuning of the varactor. The resulting $S$ parameter of Figure 6 show the antenna 

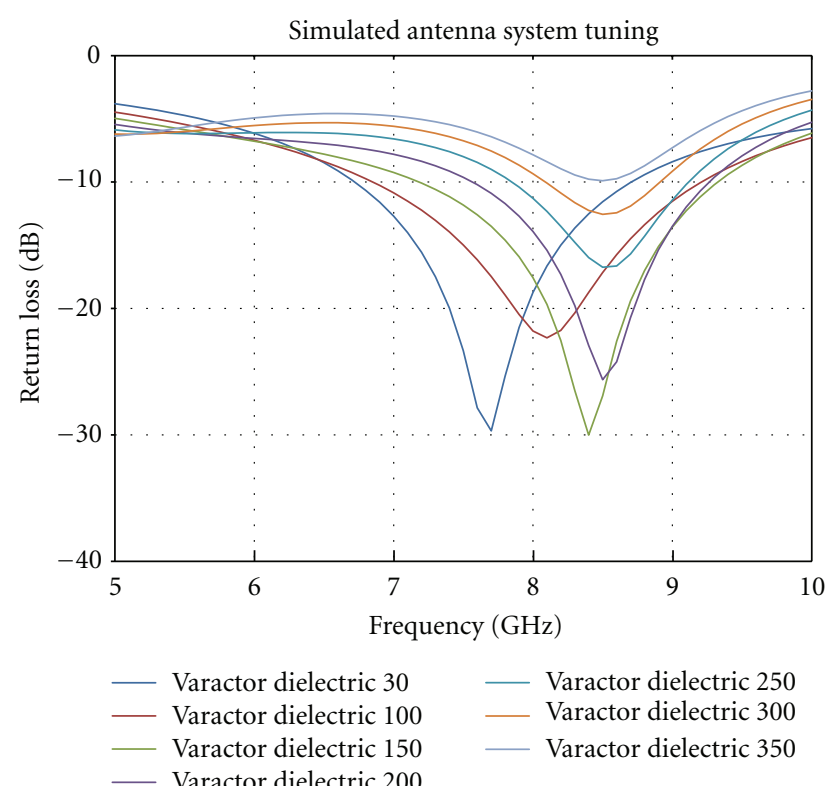

FIGURE 6: Simulated swept frequency $S_{11}$ of the antenna system.

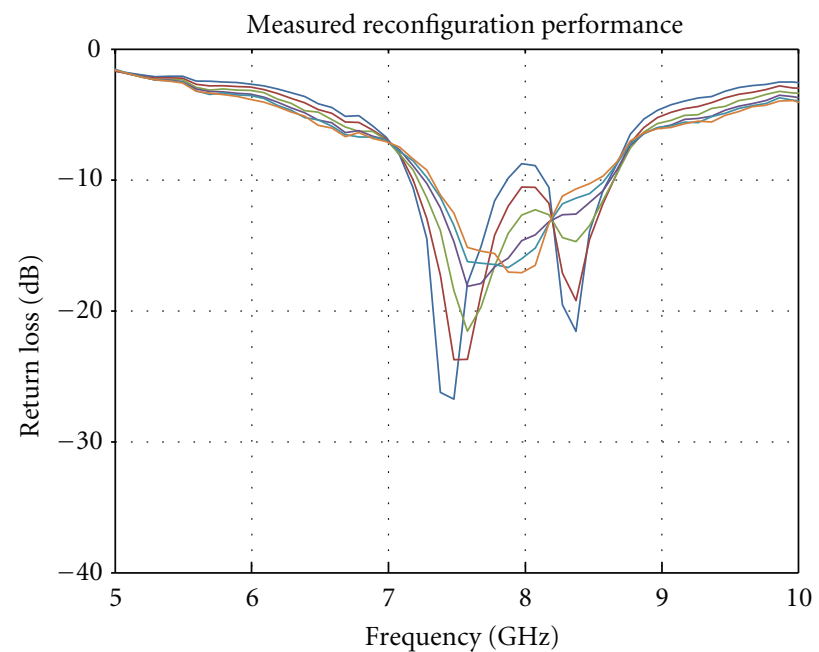

- Measured bowtie system $0 \mathrm{~V}-$ Measured bowtie system $6 \mathrm{~V}$
- Measured bowtie system $2 \mathrm{~V}-$ Measured bowtie system $8 \mathrm{~V}$
Measured bowtie system $4 \mathrm{~V}-$ Measured bowtie system $10 \mathrm{~V}$

FIgURE 7: Measured swept frequency $S_{11}$ of the antenna system under $0-10 \mathrm{~V}$ DC bias.

system tuning from 7.7 to $8.4 \mathrm{GHz}$ with altering the varactor's capacitance. As the capacitance is adjusted, both the notch points as well as the resulting $S$ parameter curves are altered. This means some bias conditions may have narrower bandwidth than others, but the overall system is now capable of operating at multiple frequencies.

The measured varactor-loaded results are shown in Figures 7 and 9. In Figure 7 we again see that the notch points and $S$ parameter curves are adjusted under differing biasing conditions. At a DC bias of $0 \mathrm{~V}$, the antenna is best matched at $7.42 \mathrm{GHz}$. This point shifts to higher frequencies as bias
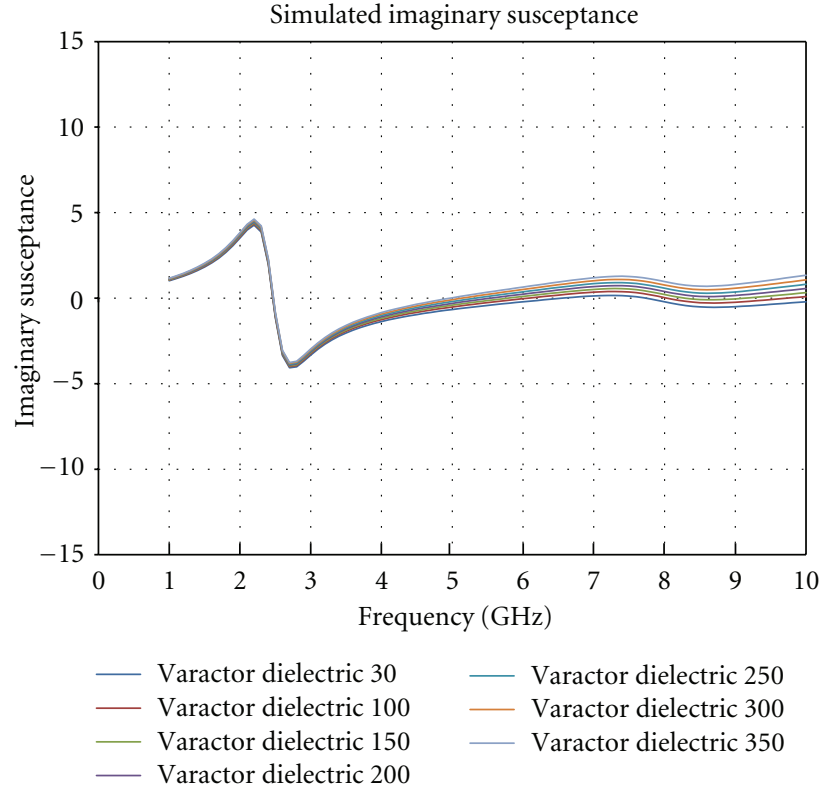

FIGURE 8: Simulated susceptance versus frequency for the antenna system.

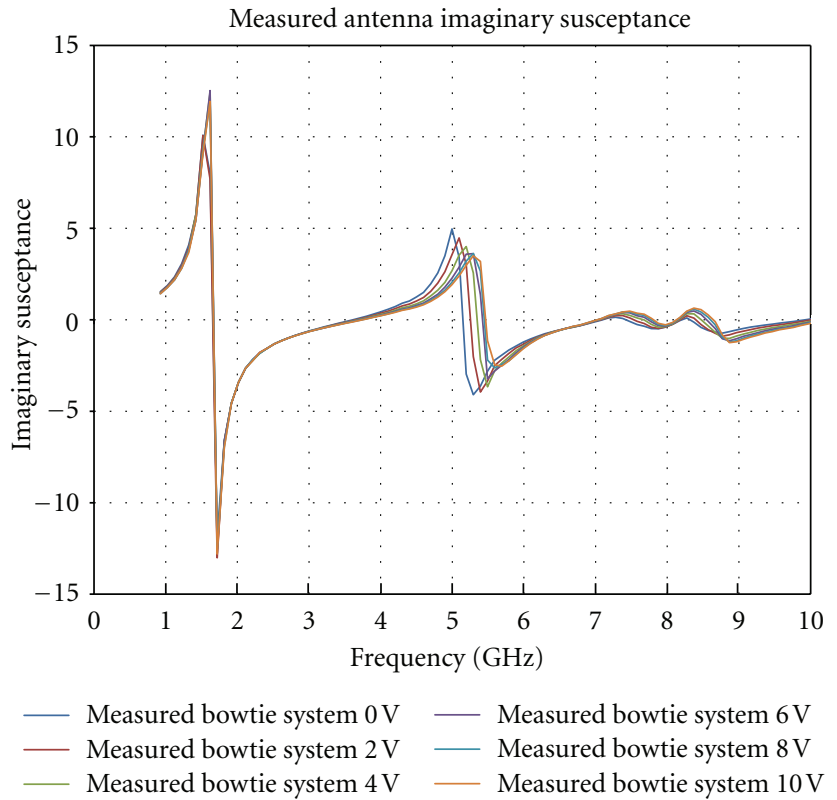

FIGURE 9: Measured susceptance versus frequency for the antenna system under 0-10 V DC bias.

voltage increases beginning to level off at bias voltages above $6 \mathrm{~V}$. The notch point shifts to $7.9 \mathrm{GHz}$ under $10 \mathrm{~V}$ bias leading to a $500 \mathrm{MHz}$ tuning of the system.

Comparing these results to the simulation plots, both the notch frequency and the overall reflection curves are different. The most noticeable difference is the appearance of the second notch at $8.36 \mathrm{GHz}$. The reasons for differing performance are under further analysis, but two conditions are 


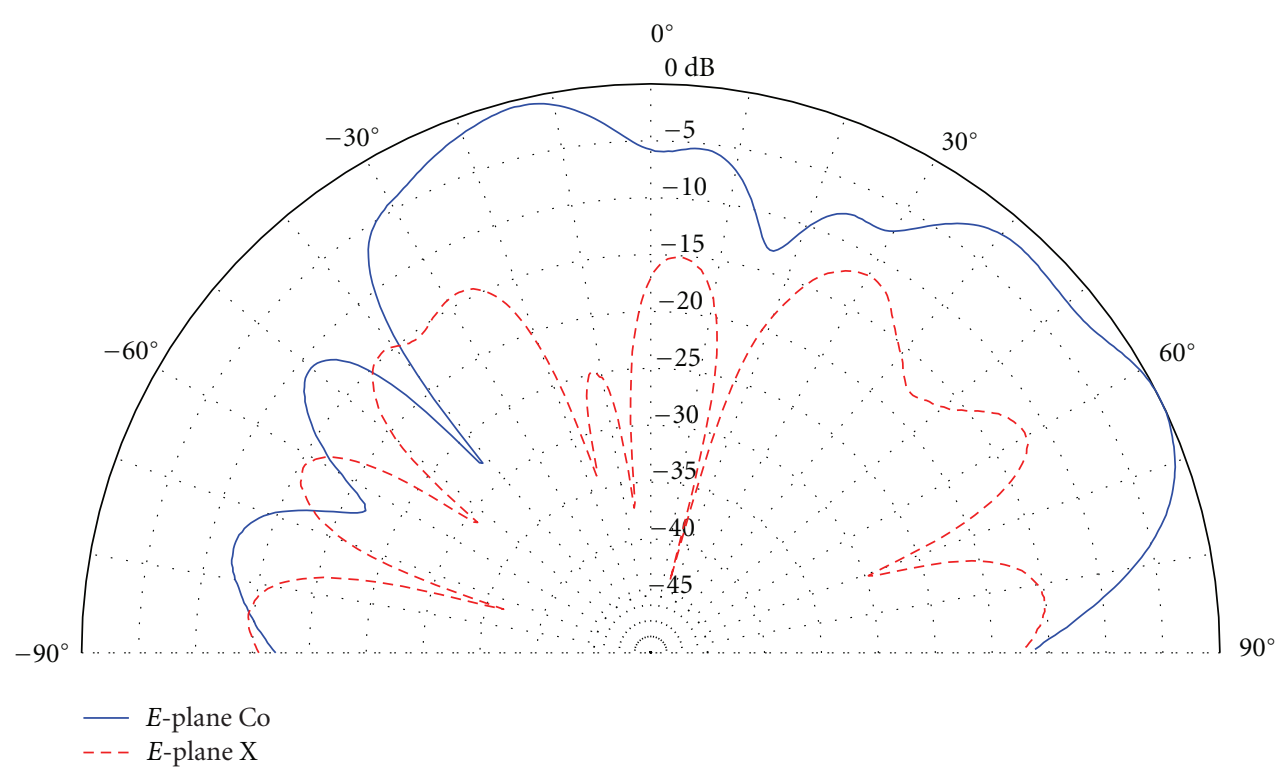

FIgURE 10: E-plane copolarization and crosspolarization measured at 7.4 GHz.

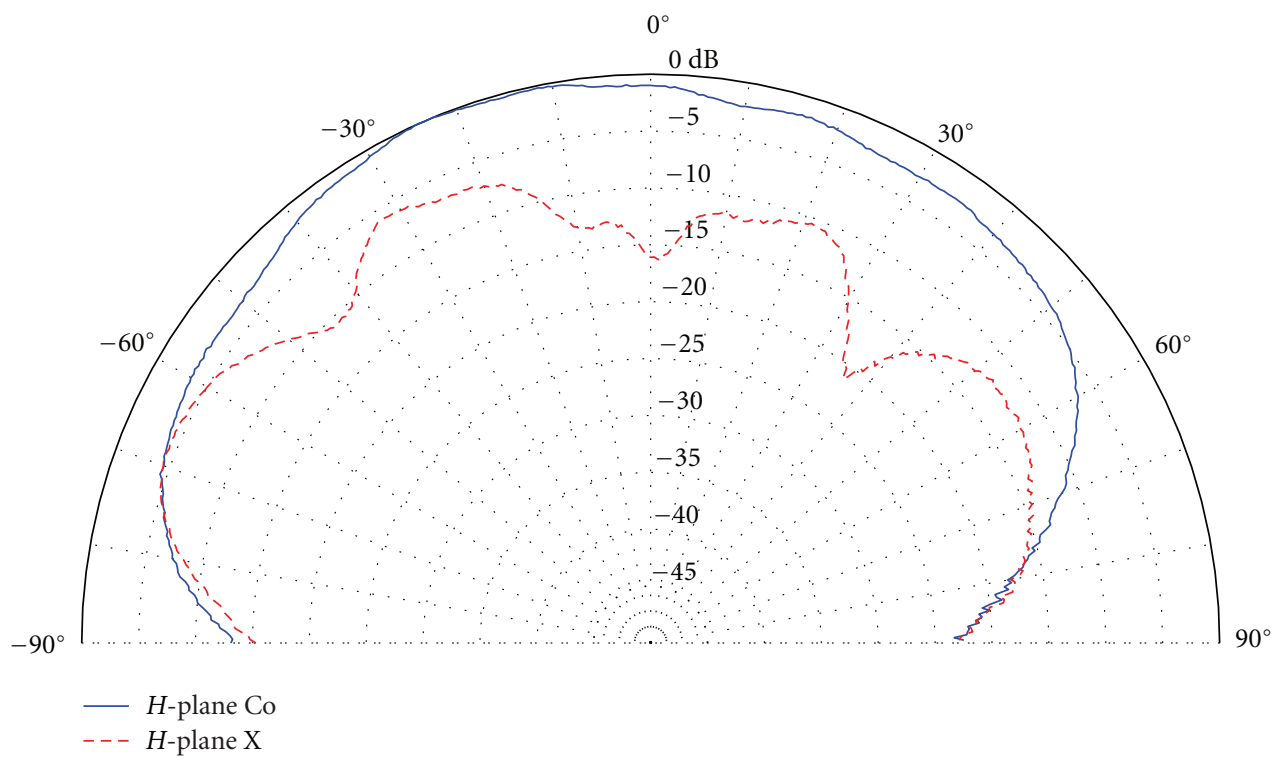

FIGURE 11: H-plane copolarization and crosspolarization measured at 7.4 GHz.

possible factors, varactor performance and BST modeling characteristics.

First the varactor performance in the system and simulation were compared by analyzing the susceptances. As can be seen in Figure 8, the addition of the shunt varactor in the simulated electromagnetic model increases the susceptance of the system across the frequency spectrum. The simulated system thus models the varactor as having very low inductance. For the measured system susceptance in Figure 9, the differences between the different bias voltages follow the same general variation up to approximately $7 \mathrm{GHz}$, at which point the curves appear to be tightly grouped. This may indicate inductive effects from the combination of the varactor and antenna that were not appropriately accounted for. This more complex susceptance near the band of interest can greatly impact the resulting system's $S$ parameter performance.

Second, the material characterization for BST in the electromagnetic AWR model was based on the performance of previous varactor devices. The model assumes a uniform $\varepsilon_{r}$ permittivity across the BST film. If either the dielectric permittivity numbers differ under the larger area of the antenna or if the permittivity is not constant over the entire film, the simulation of the antenna will be inaccurate. These possible variations in material properties in a given wafer are a definite possibility for the cause of disagreement between simulation and measurement results. 
Both copolarized and cross-polarized fields were collected for the E plane and $\mathrm{H}$ plane sweeps of the antenna system. Figures 10 and 11 show the resulting measurements at the 0 volt bias notch frequency of $7.4 \mathrm{GHz}$. From the radiation plots it is seen that the antenna does have a relatively wide beamwidth. Also, the large differences between the co-polarized and cross-polarized curves in Figures 10 and 11 indicate that the antenna operates under a highly linear polarization.

\section{Conclusions}

Improvements are needed in the correlation of electromagnetic modeling and physical performance. The combination of material and antenna characterization research will be a primary focus of future work. Investigations of additional varactor loading of antennas for improved tuning will also be of interest as well as variations to the size and type of shunt varactor used, improvements to the modeling of BST for antenna applications. As the bowtie antenna used herein is modified from the traditional structure, a more thorough analysis of the antennas mode of operation is also a point of interest.

In the end, a novel compact printed antenna for reconfigurable applications was demonstrated by employing the BST varactor in the feed network of an antenna system. This bowtie patch antenna has a compact structure with the total size of $8 \mathrm{~mm} \times 8 \mathrm{~mm}$ operating between 7 and $9 \mathrm{GHz}$. By tuning the bias voltage between 0 and $10 \mathrm{~V} \mathrm{DC}$, the notch frequency of the system is reconfigured up to $500 \mathrm{MHz}$.

\section{References}

[1] H. Jiang, M. Patterson, C. Zhang, and G. Subramanyam, "Frequency agile microstrip patch antenna using ferroelectric thin film varactor technology," in Proceedings of IEEE International Symposium on Antennas and Propagation and USNC/URSI National Radio Science Meeting (APSURSI '09), pp. 1-4, Charleston, SC, USA, June 2009.

[2] G. Subramanyam, F. Ahamed, and R. Biggers, "A Si MMIC compatible ferroelectric varactor shunt switch for microwave applications," IEEE Microwave and Wireless Components Letters, vol. 15, no. 11, pp. 739-741, 2005.

[3] E. A. Soliman, S. Brebels, G. Vandenbosch, and E. Beyne, "Xband brick wall antenna fed by CPW," Electronics Letters, vol. 34, no. 9, pp. 836-838, 1998.

[4] B. Riehl, G. Subramanyam, R. Biggers et al., "Synthesis and characterization of nanostructured BSTO thin-films for microwave applications," Integrated Ferroelectrics, vol. 55, pp. 8258379, 2003.

[5] A. L. Campbell, R. R. Biggers, G. Subramanyam et al., "Microwave characterization of nanostructured ferroelectric $\mathrm{Ba}_{0.6} \mathrm{Sr}_{0.4}$ $\mathrm{TiO}_{3}$ thin films fabricated by pulsed laser deposition," Nanotechnology, vol. 19, no. 48, Article ID 485704, 2008.

[6] H. Jiang, M. Patterson, D. Brown et al., "Miniaturized and reconfigurable CPW square-ring slot antenna using thin film varactor technology," in Proceedings of IEEE MTT-S International Microwave Symposium Digest (MTT '11), pp. 1-4, June 2011.

[7] Y. D. Lin and S. N. Tsai, "Coplanar waveguide-fed uniplanar bow-tie antenna," IEEE Transactions on Antennas and Propagation, vol. 45, no. 2, pp. 305-306, 1997. 

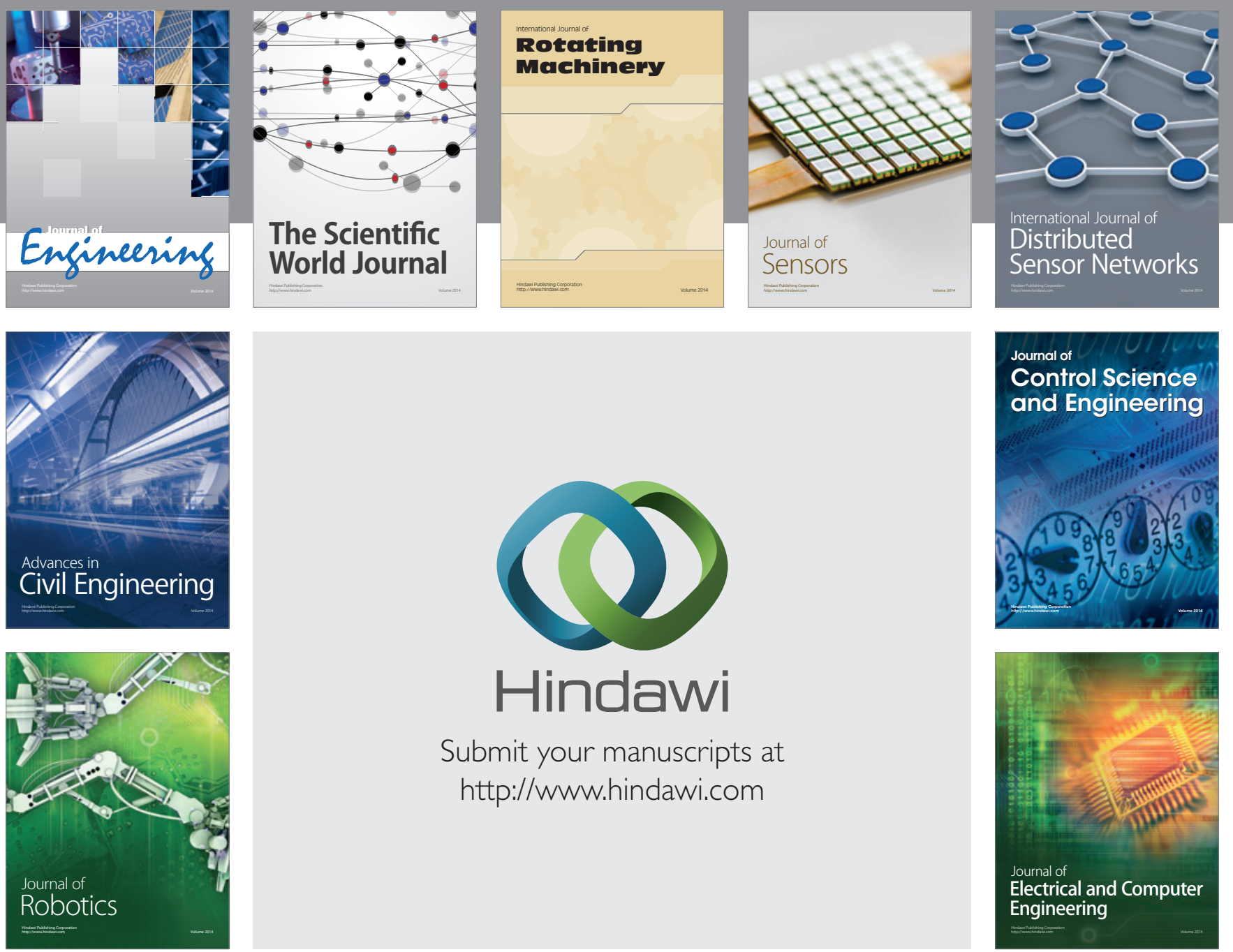

Submit your manuscripts at

http://www.hindawi.com
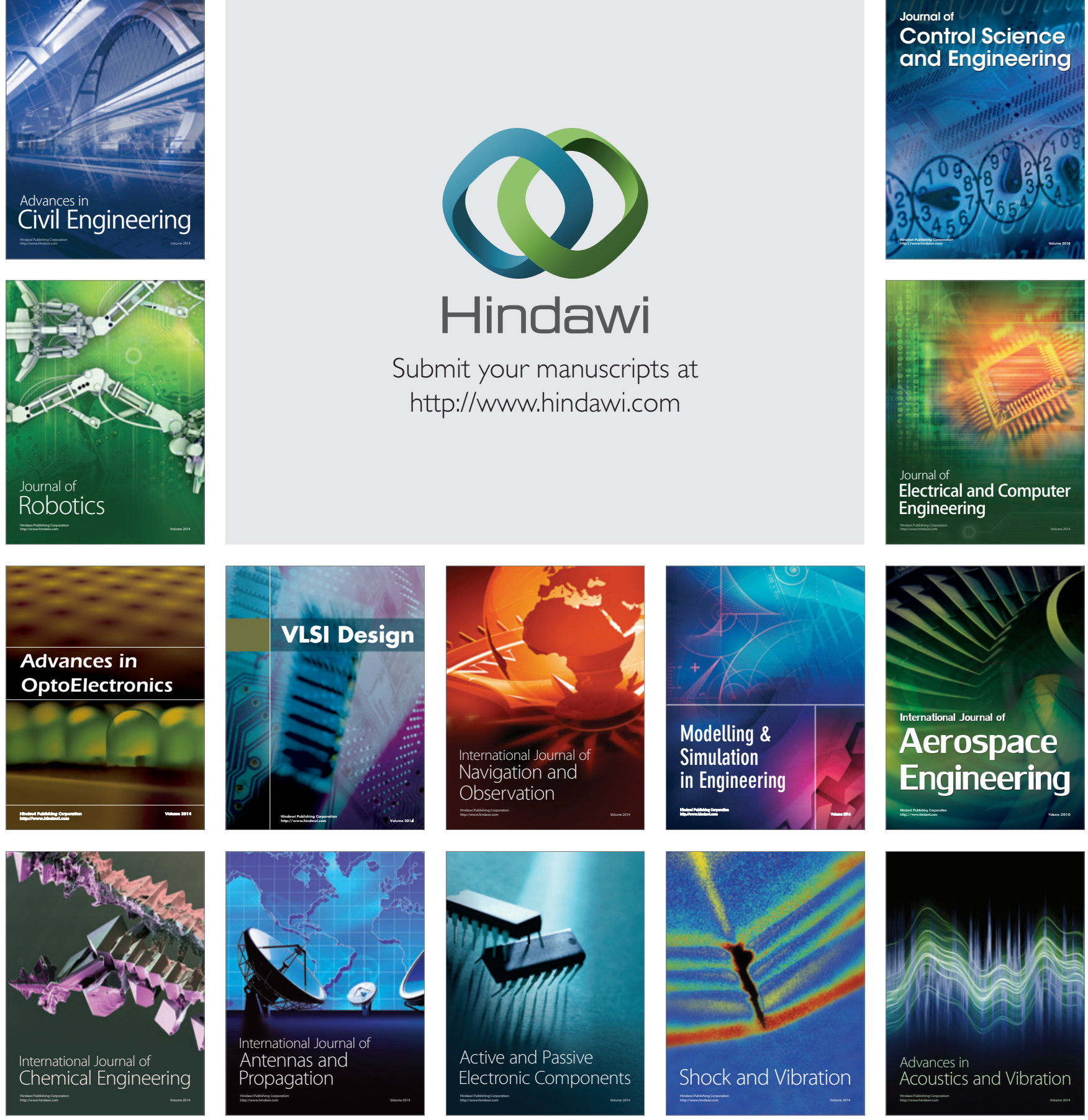